Pacific

Journal of

Mathematics

\title{
ON VOLUME GROWTH OF GRADIENT STEADY RICCI SOLITONS
}

Guofang Wei And Peng Wu

Volume $265 \quad$ No. 1

September 2013 


\title{
ON VOLUME GROWTH OF GRADIENT STEADY RICCI SOLITONS
}

\author{
Guofang Wei And Peng Wu
}

In this paper we study volume growth of gradient steady Ricci solitons. We show that if the potential function satisfies a uniform condition, then the soliton has at most euclidean volume growth.

\section{Introduction}

$\left(M^{n}, g\right)$ is a gradient Ricci soliton if there is a smooth function $f: M \rightarrow \mathbb{R}$ and constant $\lambda \in \mathbb{R}$ such that

$$
\text { Ric }+ \text { Hess } f=\lambda g .
$$

We refer to $f$ as the potential function. The soliton is called shrinking, steady, and expanding when $\lambda>0, \lambda=0$, and $\lambda<0$ respectively.

Ricci solitons are self-similar solutions of the Ricci flow, and play an important role in the study of singularity formation. They are also natural extensions of Einstein manifolds, and special cases of smooth metric measure spaces.

Volume growth of gradient Ricci solitons is of particular interest to mathematicians. Estimates of the potential functions plays an important role in the study of volume growth. Hamilton [1995] proved the following identity for gradient Ricci solitons:

$$
R+|\nabla f|^{2}-2 \lambda f=\Lambda,
$$

where $\Lambda$ is a constant, and $R$ is the scalar curvature.

For gradient shrinking Ricci solitons, the answer is complete. Perelman [2003] and Cao and Zhou [2010] proved that $f$ always grows quadratically. Cao and Zhou [2010] further proved that any gradient Ricci shrinking soliton has at most euclidean volume growth. Recently, Munteanu and Wang [2012] proved that any noncompact gradient Ricci shrinking soliton has at least linear volume growth.

For gradient steady Ricci solitons, B. L. Chen [2009] proved that $R \geq 0$. Hence, $\Lambda \geq 0$, and equal to zero if and only if $f$ is constant and $(M, g)$ is Ricci flat. When

Keywords: Ricci solitons, volume growth. 
$\Lambda>0$ we can assume $\Lambda=1$ after scaling; that is,

$$
R+|\nabla f|^{2}=1 .
$$

Combined with the trace of the steady Ricci soliton equation $R+\Delta f=0$, we have

$$
\Delta f-|\nabla f|^{2}=-1 \text {. }
$$

Therefore, $f$ has no local minimum. Equation (1-2) and $R \geq 0$ give $|\nabla f| \leq 1$. Namely, $f$ decays at most linearly.

Cao and Chen [2012] proved that $f$ decays linearly when Ricci curvature is positive, and $R$ attains its maximum at some point. However, the simple example of $\mathbb{R}^{2}$ with the canonical metric $g_{0}$ and $f(x)=x_{1}$ shows that this is not the case; $f$ is constant along the $x_{2}$ direction. Note that the Riemannian product of any two steady gradient Ricci solitons is still a steady gradient Ricci soliton. Hence, a steady gradient Ricci soliton multiplied with a trivial one ( $f$ is a constant) will have constant direction. Though one can take the product of two shrinking ones, all trivial shrinking ones are compact, so they will not give a constant direction by taking a product. Munteanu and Sesum [2013] and Wu [2013] independently showed that the infimum of $f$ does decay linearly. In fact,

$$
-r \leq \inf _{y \in \partial B(x, r)} f(y)-f(x) \leq-r+\sqrt{2 n}(\sqrt{r}+1), \quad r \gg 1 .
$$

In particular, $\liminf \operatorname{in}_{y \rightarrow \infty} R(y)=0$. See also [Fernández-López and García-Río 2011; Chow et al. 2011].

We note that among all known examples of steady gradient Ricci solitons, the infimum of $f$ is like $-r+O(\ln r)$. See the survey article [Cao 2010] for a list of examples. One naturally asks if one can improve the second order term in (1-4) from $\sqrt{r}$ to $\ln r$. We show this is indeed the case for a large class of steady gradient Ricci solitons. To study the second order term, write the potential function in polar coordinates:

$$
f(r, \theta)=-r+\phi(r, \theta),
$$

where $r(\cdot)=d(x, \cdot)$ for some $x \in M^{n}, \theta \in S^{n-1}$. Without loss of generality, we assume $\phi(0, \theta)=0$ by adding a constant to $f$. Since $|\nabla f| \leq 1$, we have $|\partial f / \partial r| \leq 1$, so $f(r) \geq-r, \phi(r) \geq 0$ and $\phi(r, \theta)$ are nondecreasing in $r$ for any fixed $\theta$. We show that the estimate (1-4) can be improved to $\ln r$ if $\phi$ in one direction is comparable to the minimum of $\phi$ among all spherical directions for all large $r$.

Theorem 1.1. Let $\left(M^{n}, g, f\right)$ be a complete gradient steady Ricci soliton satisfying (1-2). Assume that there exist $\theta_{0} \in S^{n-1}$, and constants $C_{1} \geq 0, C_{2} \geq 0$ such that

$$
\int_{0}^{r}\left(\phi\left(r, \theta_{0}\right)-\phi\left(t, \theta_{0}\right)\right) d t \leq C_{1} \min _{\theta \in S^{n-1}} \int_{0}^{r}(\phi(r, \theta)-\phi(t, \theta)) d t+C_{2} r
$$


for sufficiently large $r$. Then for any $x \in M^{n}$, there exist constants $C \geq 0$ and $r_{0}>0$ such that for $r \geq r_{0}$,

$$
-r \leq \inf _{y \in \partial B(x, r)} f(y)-f(x) \leq-r+\left(\frac{n}{2} C_{1}+C_{2}\right) \ln r+C .
$$

Remark 1.2. All known examples of gradient steady Ricci solitons satisfy the condition (1-5). We suspect that the estimate (1-6) holds for all gradient steady Ricci solitons.

In [Munteanu and Sesum 2013], it was proven that any gradient steady Ricci soliton has at least linear volume growth, and at most a growth rate of $e^{\sqrt{r}}$. We show that if the potential function satisfies a uniform condition in the spherical directions, then the gradient steady Ricci soliton has at most euclidean volume growth.

Theorem 1.3. Let $\left(M^{n}, g, f\right)$ be a complete gradient steady Ricci soliton satisfying (1-2). Assume that there exist constants $C_{1}, C_{2} \geq 0$ such that

(1-7) $\max _{\theta \in S^{n-1}} \int_{0}^{r}(\phi(r, \theta)-\phi(t, \theta)) d t \leq C_{1} \min _{\theta \in S^{n-1}} \int_{0}^{r}(\phi(r, \theta)-\phi(t, \theta)) d t+C_{2} r$

for sufficiently large $r$. Then for any $x \in M^{n}$, there exist constants $C \geq 0$ and $r_{0}>0$ such that for any $r \geq r_{0}$,

$$
-r \leq f(y)-f(x) \leq-r+C \ln r
$$

for any $y \in \partial B(x, r)$. Moreover, the soliton has at most euclidean volume growth; that is, for any $x \in M^{n}$ there exists $r_{0}>0$, and for any $r \geq r_{0}$,

$$
\operatorname{Vol} B(x, r) \leq C r^{n} \text {. }
$$

If , in addition, $\phi(r) \geq \delta \ln r$ for large $r$, then

$$
\operatorname{Vol} B(x, r) \leq C r^{n-\delta} .
$$

Remark 1.4. (1) If $\phi$ increases uniformly along all spherical directions; that is, $\max _{\theta} \partial \phi / \partial r \leq C \min _{\theta} \partial \phi / \partial r$, where $\theta \in S^{n-1}$, then $\phi$ satisfies (1-7) with $C_{1}=C$ and $C_{2}=0$.

(2) Theorem 1.3 can be considered an analogue of the volume growth theorem of [Cao and Zhou 2010], valid for gradient shrinking Ricci solitons. As the potential function for such solitons automatically satisfies a uniform condition, here too we need to impose a uniform condition for gradient steady Ricci solitons.

(3) If the soliton is rectifiable (see [Petersen and Wylie 2009]) - i.e., $f$ is the distance function from a set - then $\phi$ satisfies (1-7) with $C_{1}=1$ if the set is bounded (this is the case with all nonproduct examples). 
To prove the results, the following estimate for $\phi$, which holds for all gradient steady Ricci solitons, is the key:

Proposition 1.5. Let $\left(M^{n}, g, f\right)$ be a complete gradient steady Ricci soliton satisfying (1-2). Then

$$
\min _{y \in \partial B(x, r)} \int_{0}^{r}(\phi(y)-\phi(t)) d t \leq \frac{n}{2}(r+\sqrt{r})+o\left(\frac{1}{r}\right) .
$$

This estimate improves the one in [Wu 2013]. In the next section we derive a volume comparison for the solitons by adapting the volume comparison for smooth metric measure spaces in [Wei and Wylie 2009]. Then we prove Proposition 1.5 by combining this with (1-3). In Section 3 we prove the main theorems using this estimate and an ODE.

\section{The preliminary estimate}

In this section we prove Proposition 1.5 by applying a weighted volume comparison argument for smooth metric measure spaces as in [Wei and Wylie 2009; Wu 2013].

Recall that a smooth metric measure space is a triple $\left(M^{n}, g, e^{-f} d \operatorname{vol}_{g}\right)$, where $\left(M^{n}, g\right)$ is a smooth Riemannian manifold, and $f: M^{n} \rightarrow \mathbb{R}$ is a smooth function. Write the volume element in polar coordinates $d \mathrm{vol}=J(r, \theta) d r d \theta$. Define the weighted volume element as $J_{f}(r, \theta)=e^{-f} J(r, \theta)$ and the weighted volume as $\operatorname{Vol}_{f} B(x, r)=\int_{B(x, r)} e^{-f} d$ vol.

Wei and Wylie [2009] obtained the following $f$-volume comparison theorem for smooth metric measure spaces:

Theorem 2.1 ( $f$-volume comparison). Suppose $\left(M^{n}, g, e^{-f} d\right.$ vol) is a smooth metric measure space with Ric $_{f} \geq(n-1) H$. Fix $x \in M$. If $|f| \leq \Lambda$, then for $R \geq r>0($ and $R \leq \pi / 4 \sqrt{H}$ if $H>0)$,

$$
\frac{\operatorname{Vol}_{f} B(x, R)}{\operatorname{Vol}_{f} B(x, r)} \leq \frac{V_{H}^{n+4 \Lambda}\left(B_{R}\right)}{V_{H}^{n+4 \Lambda}\left(B_{r}\right)}
$$

where $V_{H}^{n}\left(B_{r}\right)$ is the volume of the ball of radius $r$ in $M_{H}^{n}$ (the simply connected model space of dimension $n$ with constant sectional curvature $H$ ).

One observes that the dimension of the model space in the volume comparison depends on the potential function $f$. Further investigation of the dimension will lead to Proposition 1.5.

Denote the $f$-mean curvature by $m_{f}=\left(\ln J_{f}\right)^{\prime}$. For $0<r_{1} \leq r_{2}$, let $A\left(x, r_{1}, r_{2}\right)=$ $\left\{y \mid r_{1} \leq d(x, y) \leq r_{2}\right\}$ be the annulus, and

$$
a=\min _{y \in A\left(x, r_{1}, r_{2}\right)} \frac{2}{r(y)^{2}} \int_{0}^{r(y)}(\phi(y)-\phi(t)) d t .
$$


Clearly $a \geq 0$. By (1-4), we have $a \leq C / \sqrt{r_{1}}$ for $r_{1} \gg 1$. For the rest we assume $r_{1} \gg 1$ and therefore we can assume $a<1$.

Proposition 2.2. For a gradient steady Ricci soliton, we have

$$
\begin{aligned}
m_{f}(r, \theta) \leq & \frac{n-1}{r}+1-\frac{2}{r^{2}} \int_{0}^{r}(\phi(r, \theta)-\phi(t, \theta)) d t \leq \frac{n-1}{r}+1, \\
& \frac{\operatorname{Vol}_{f} \partial B\left(x, r_{2}\right)}{\operatorname{Vol}_{f} A\left(x, r_{1}, r_{2}\right)} \leq \frac{n / r_{2}+1-a}{1-\left(r_{1} / r_{2}\right)^{n+(1-a) r_{2}}} .
\end{aligned}
$$

Proof. For a smooth metric space $\left(M^{n}, g, f\right)$ with Ric $_{f} \geq 0$, recall the following estimate for $m_{f}$ from [Wei and Wylie 2009, (3.19)]:

$$
m_{f}(r, \theta) \leq \frac{n-1}{r}+\frac{2}{r^{2}} \int_{0}^{r}(f(t)-f(r)) d t .
$$

Plugging in $f=-r+\phi$ gives (2-1).

Now let

$$
\bar{m}(r)= \begin{cases}\frac{n-1}{r}+1 & \text { if } r \leq r_{1}, \\ \frac{n-1+(1-a) r_{2}}{r} & \text { if } r_{1}<r \leq r_{2} .\end{cases}
$$

Then

$$
m_{f}(r) \leq \bar{m}(r) \text { for } 0<r \leq r_{2} .
$$

Let $\bar{A}(r)=e^{\int_{0}^{r} \bar{m}(t) d t}$ and $\bar{V}\left(r_{0}, r\right)=\int_{r_{0}}^{r} \bar{A}(t) d t$. From the mean curvature relation (2-3), we have $\left(A_{f} / \bar{A}\right)^{\prime} \leq 0$; therefore

$$
\frac{\operatorname{Vol}_{f} \partial B\left(x, r_{2}\right)}{\operatorname{Vol}_{f} A\left(x, r_{1}, r_{2}\right)} \leq \frac{\bar{A}\left(r_{2}\right)}{\bar{V}\left(r_{1}, r_{2}\right)} .
$$

We compute

$$
\begin{aligned}
\frac{\bar{A}\left(r_{2}\right)}{\bar{V}\left(r_{1}, r_{2}\right)} & =\frac{e^{\int_{0}^{r_{2}} \bar{m}(t) d t}}{\int_{r_{1}}^{r_{2}} e^{\int_{0}^{s} \bar{m}(t) d t} d s}=\frac{e^{\int_{r_{1}}^{r_{2}} \bar{m}(t) d t}}{\int_{r_{1}}^{r_{2}} e^{\int_{r_{1}}^{s} \bar{m}(t) d t} d s} \\
& =\frac{\left(r_{2} / r_{1}\right)^{n-1+(1-a) r_{2}}}{\int_{r_{1}}^{r_{2}}\left(s / r_{1}\right)^{n-1+(1-a) r_{2}} d s}=\frac{n / r_{2}+1-a}{1-\left(r_{1} / r_{2}\right)^{n+(1-a) r_{2}}} .
\end{aligned}
$$

This gives (2-2).

Proof of Proposition 1.5. Integrating (1-3) and using $|\nabla f| \leq 1$ we have, for any $x \in M$,

$$
\begin{aligned}
\int_{B(x, r)} 1 \cdot e^{-f} d \mathrm{vol} & =-\int_{B(x, r)}\left(\Delta f-|\nabla f|^{2}\right) \cdot e^{-f} d \mathrm{vol} \\
& =-\int_{\partial B(x, r)} \frac{\partial f}{\partial n} e^{-f} d \mathrm{vol} \leq \int_{\partial B(x, r)} e^{-f} d \mathrm{vol} .
\end{aligned}
$$


Therefore,

$$
\frac{\operatorname{Vol}_{f} \partial B(x, r)}{\operatorname{Vol}_{f} B(x, r)} \geq 1 .
$$

Combining (2-2) and (2-4) we have

$$
a \leq \frac{n}{r_{2}}+\left(\frac{r_{1}}{r_{2}}\right)^{n+(1-a) r_{2}} .
$$

Let $r_{1}=r$ and $r_{2}=r+\sqrt{r}$. Then $r_{1} / r_{2}=(1+1 / \sqrt{r})^{-1}$. When $r$ is large,

$$
\left(1+\frac{1}{\sqrt{r}}\right)^{-(n+(1-a)(r+\sqrt{r}))}=O\left(e^{-(1-a) \sqrt{r}}\right) .
$$

Therefore, for all $r$ large enough,

$$
a=\min _{y \in A(x, r, r+\sqrt{r})} \frac{2}{r(y)^{2}} \int_{0}^{r(y)}(\phi(y)-\phi(t)) d t \leq \frac{n}{r+\sqrt{r}}+O\left(e^{-(1-a) \sqrt{r}}\right) .
$$

Suppose the minimum above is attained at $y_{0}=\left(r_{0}, \theta_{1}\right)$ with $r \leq r_{0} \leq r+\sqrt{r}$. Then

$$
\begin{aligned}
\min _{y \in \partial B(x, r)} \int_{0}^{r}(\phi(y)-\phi(t)) d t & \leq \int_{0}^{r_{0}}\left(\phi\left(y_{0}\right)-\phi(t)\right) d t \\
& \leq \frac{r_{0}^{2}}{2}\left(\frac{n}{r+\sqrt{r}}+O\left(e^{-(1-a) \sqrt{r}}\right)\right) \\
& \leq \frac{n}{2}(r+\sqrt{r})+o\left(\frac{1}{r}\right) .
\end{aligned}
$$

\section{Proof of main results}

Proof of Theorem 1.1. From (1-9) and (1-5), we have

$$
\int_{0}^{r}\left(\phi\left(r, \theta_{0}\right)-\phi\left(t, \theta_{0}\right)\right) d t \leq \frac{n C_{1}}{2}(r+\sqrt{r})+C_{2} r+o\left(\frac{1}{r}\right) .
$$

For simplicity, when there is no confusion we omit $\theta_{0}$ in the function. Let $\Phi(r)=$ $\int_{0}^{r} \phi(t) d t$; then (3-1) can be written as

$$
\Phi^{\prime}(r)-\frac{1}{r} \Phi(r) \leq \frac{n C_{1}}{2}+C_{2}+O\left(\frac{1}{\sqrt{r}}\right) .
$$

Multiplying by the integrating factor $1 / r$ and integrating from some fixed $t_{0} \gg 1$ to $r$, we get

$$
\frac{\Phi(r)}{r} \leq\left(\frac{n C_{1}}{2}+C_{2}\right) \ln r+C_{3}
$$


So, we have

$$
\begin{aligned}
\phi\left(r, \theta_{0}\right) & =\Phi^{\prime}\left(r, \theta_{0}\right) \leq \frac{\Phi\left(r, \theta_{0}\right)}{r}+\frac{n C_{1}}{2}+C_{2}+O\left(\frac{1}{\sqrt{r}}\right) \\
& \leq\left(\frac{n C_{1}}{2}+C_{2}\right) \ln r+C_{4} \\
f\left(r, \theta_{0}\right) & =-r+\phi\left(r, \theta_{0}\right) \leq-r+\left(\frac{n C_{1}}{2}+C_{2}\right) \ln r+C_{4} .
\end{aligned}
$$

This gives (1-6).

Proof of Theorem 1.3. From (1-9) and (1-7), we have

$$
\int_{0}^{r}(\phi(r, \theta)-\phi(t, \theta)) d t \leq \frac{n C_{1}}{2}(r+\sqrt{r})+C_{2} r+o\left(\frac{1}{r}\right)
$$

for all $\theta \in S^{n-1}$. Therefore, (1-6) holds for all $y$. Namely, for all $y \in \partial B(x, r)$,

$$
-r \leq f(y)-f(x) \leq-r+\left(\frac{n C_{1}}{2}+C_{2}\right) \ln r+C_{4} .
$$

By (2-1), for all $r>0$,

$$
\begin{aligned}
\frac{\partial}{\partial r} \ln J & =m_{f}(r)+\langle\nabla f, \nabla r\rangle \\
& \leq \frac{n-1}{r}+1-\frac{2}{r} \phi(r)+\frac{2}{r^{2}} \int_{0}^{r} \phi(t) d t+\langle\nabla f, \nabla r\rangle .
\end{aligned}
$$

Integrating from 1 to $r$ and performing integration by parts for the double integral, we get

(3-3) $\quad \ln J(r)-\ln J(1)$

$$
\begin{aligned}
& \leq(n-1) \ln r+(r-1)-\int_{1}^{r} \frac{2}{s} \phi(s) d s+\left.\left(-\frac{2}{s} \int_{0}^{s} \phi(t) d t\right)\right|_{1} ^{r} \\
&+\int_{1}^{r} \frac{2}{s} \phi(s) d s+f(r)-f(1) \\
&=(n-1) \ln r+\phi(r)-\frac{2}{r} \int_{0}^{r} \phi(t) d t+2 \int_{0}^{1} \phi(t) d t-f(1)-1 \\
&=(n-1) \ln r-\phi(r)+2\left(\phi(r)-\frac{1}{r} \int_{0}^{r} \phi(t) d t\right)+2 \int_{0}^{1} \phi(t) d t-f(1)-1 .
\end{aligned}
$$

Using (3-2) we have, for large $r$,

$$
\ln J(r) \leq(n-1) \ln r-\phi(r)+C \leq(n-1) \ln r+C .
$$

Hence,

$$
J(r) \leq e^{C} e^{(n-1) \ln r}=e^{C} r^{n-1},
$$


and the volume of a geodesic ball centered at $x$ satisfies

$$
\operatorname{Vol} B(x, r) \leq C^{\prime} r^{n}
$$

If further $\phi(s) \geq \delta \ln s$, then we have

$$
J(r) \leq C r^{n-1} \exp (-\phi(r)) \leq C r^{n-\delta-1},
$$

therefore the volume growth is strictly less than euclidean volume growth:

$$
\operatorname{Vol} B(x, r) \leq C r^{n-\delta} .
$$

For general gradient steady Ricci solitons, the estimate of a potential function can be reduced to the following:

Question 3.1. Suppose $\phi$ is nondecreasing along any minimal geodesic starting from $x$. Assume that for sufficiently large $r, \inf _{y \in \partial B(x, r)} \phi(y) \leq C \sqrt{r}$, and

$$
\inf _{y \in \partial B(x, r)} \int_{1}^{r}\left(\phi(y)-\phi\left(\gamma_{y}(t)\right) d t \leq \frac{n r}{2} .\right.
$$

Does the following hold?

$$
\inf _{y \in \partial B(x, r)} \phi(y) \leq C \ln r
$$

Remark 3.2. From (3-3), we see that if

$$
-r \leq f(y)-f(x) \leq-r+C \ln r
$$

for $y \in \partial B(x, r)$, then for any $x \in M^{n}$, there exists $r_{0}>0$ such that for any $r \geq r_{0}$,

$$
\operatorname{Vol} B(x, r) \leq C^{\prime} r^{n+C} .
$$

\section{Acknowledgements}

The second author would like to thank Professor Thomas Sideris for helpful discussions.

\section{References}

[Cao 2010] H.-D. Cao, "Recent progress on Ricci solitons", pp. 1-38 in Recent advances in geometric analysis (Taipei, 2007), edited by Y.-I. Lee et al., Adv. Lect. Math. 11, International Press, Somerville, MA, 2010. MR 2011d:53061 Zbl 1201.53046 arXiv 0908.2006

[Cao and Chen 2012] H.-D. Cao and Q. Chen, "On locally conformally flat gradient steady Ricci solitons”, Trans. Amer. Math. Soc. 364:5 (2012), 2377-2391. MR 2888210 Zbl 1245.53038

[Cao and Zhou 2010] H.-D. Cao and D. Zhou, "On complete gradient shrinking Ricci solitons", J. Differential Geom. 85:2 (2010), 175-185. MR 2011k:53040 Zbl 1246.53051

[Chen 2009] B.-L. Chen, "Strong uniqueness of the Ricci flow", J. Differential Geom. 82:2 (2009), 363-382. MR 2010h:53095 Zbl 1177.53036 
[Chow et al. 2011] B. Chow, P. Lu, and B. Yang, "Lower bounds for the scalar curvatures of noncompact gradient Ricci solitons", C. R. Math. Acad. Sci. Paris 349:23-24 (2011), 1265-1267. MR 2861997 Zbl 1230.53036

[Fernández-López and García-Río 2011] M. Fernández-López and E. García-Río, "Maximum principles and gradient Ricci solitons", J. Differential Equations 251:1 (2011), 73-81. MR 2012d:53136 Zbl 1217.53042

[Hamilton 1995] R. S. Hamilton, "The formation of singularities in the Ricci flow", pp. 7-136 in Proceedings of the conference on geometry and topology (Cambridge, MA, 1993), edited by C. C. Hsiung and S.-T. Yau, Surveys in Differential Geometry 2, International Press, Somerville, MA, 1995. MR 97e:53075 Zbl 0867.53030

[Munteanu and Sesum 2013] O. Munteanu and N. Sesum, "On gradient Ricci solitons", J. Geom. Anal. 23:2 (2013), 539-561. MR 3023848

[Munteanu and Wang 2012] O. Munteanu and J. Wang, "Analysis of weighted Laplacian and applications to Ricci solitons”, Comm. Anal. Geom. 20:1 (2012), 55-94. MR 2903101 Zbl 1245.53039 arXiv 1112.3027

[Perelman 2003] G. Y. Perelman, "Ricci flow with surgery on three manifolds", preprint, 2003. Zbl 1130.53002 arXiv math.DG/0303109

[Petersen and Wylie 2009] P. Petersen and W. Wylie, "On gradient Ricci solitons with symmetry", Proc. Amer. Math. Soc. 137:6 (2009), 2085-2092. MR 2010a:53073 Zbl 1168.53021

[Wei and Wylie 2009] G. Wei and W. Wylie, "Comparison geometry for the Bakry-Emery Ricci tensor”, J. Differential Geom. 83:2 (2009), 377-405. MR 2011a:53064 Zbl 1189.53036

[Wu 2013] P. Wu, "On the potential function of gradient steady Ricci solitons", J. Geom. Anal. 23:1 (2013), 221-228. MR 3010278 Zbl 06136841

Received July 12, 2012. Revised November 9, 2012.

GUOFANG WeI

Math Department

UC SANTA BARBARA

SANTA BARBARA, CA 93106

UNITED STATES

wei@math.ucsb.edu

PENG Wu

Math Department

CORNELl UNIVERSITY

ITHACA, NY 14853

UNITED STATES

wupenguin@math.cornell.edu 


\title{
PACIFIC JOURNAL OF MATHEMATICS
}

\author{
msp.org/pjm
}

Founded in 1951 by E. F. Beckenbach (1906-1982) and F. Wolf (1904-1989)

\section{EDITORS}

V. S. Varadarajan (Managing Editor)

Department of Mathematics

University of California

Los Angeles, CA 90095-1555

pacific@math.ucla.edu

Paul Balmer

Department of Mathematics

University of California

Los Angeles, CA 90095-1555

balmer@math.ucla.edu

Daryl Cooper

Department of Mathematics

University of California

Santa Barbara, CA 93106-3080 cooper@math.ucsb.edu

Jiang-Hua $\mathrm{Lu}$

Department of Mathematics

Pokfulam Rd., Hong Kong jhlu@maths.hku.hk
The University of Hong Kong

Don Blasius

Department of Mathematics University of California

Los Angeles, CA 90095-1555

blasius@math.ucla.edu

Robert Finn

Department of Mathematics Stanford University

Stanford, CA 94305-2125

finn@math.stanford.edu

Sorin Popa

Department of Mathematics

University of California

Los Angeles, CA 90095-1555 popa@math.ucla.edu

Paul Yang

Department of Mathematics Princeton University

Princeton NJ 08544-1000

yang@math.princeton.edu

\section{PRODUCTION}

Silvio Levy, Scientific Editor, production@msp.org

\section{SUPPORTING INSTITUTIONS}

ACADEMIA SINICA, TAIPEI

CALIFORNIA INST. OF TECHNOLOGY

INST. DE MATEMÁTICA PURA E APLICADA

KEIO UNIVERSITY

MATH. SCIENCES RESEARCH INSTITUTE

NEW MEXICO STATE UNIV.

OREGON STATE UNIV.

\author{
STANFORD UNIVERSITY \\ UNIV. OF BRITISH COLUMBIA \\ UNIV. OF CALIFORNIA, BERKELEY \\ UNIV. OF CALIFORNIA, DAVIS \\ UNIV. OF CALIFORNIA, LOS ANGELES \\ UNIV. OF CALIFORNIA, RIVERSIDE \\ UNIV. OF CALIFORNIA, SAN DIEGO \\ UNIV. OF CALIF., SANTA BARBARA
}

\author{
Vyjayanthi Chari \\ Department of Mathematics \\ University of California \\ Riverside, CA 92521-0135 \\ chari@math.ucr.edu \\ Kefeng Liu \\ Department of Mathematics \\ University of California \\ Los Angeles, CA 90095-1555 \\ liu@math.ucla.edu \\ Jie Qing \\ Department of Mathematics \\ University of California \\ Santa Cruz, CA 95064 \\ qing@cats.ucsc.edu
}

These supporting institutions contribute to the cost of publication of this Journal, but they are not owners or publishers and have no responsibility for its contents or policies.

See inside back cover or msp.org/pjm for submission instructions.

The subscription price for 2013 is US \$400/year for the electronic version, and \$485/year for print and electronic.

Subscriptions, requests for back issues and changes of subscribers address should be sent to Pacific Journal of Mathematics, P.O. Box 4163, Berkeley, CA 94704-0163, U.S.A. The Pacific Journal of Mathematics is indexed by Mathematical Reviews, Zentralblatt MATH, PASCAL CNRS Index, Referativnyi Zhurnal, Current Mathematical Publications and the Science Citation Index.

The Pacific Journal of Mathematics (ISSN 0030-8730) at the University of California, c/o Department of Mathematics, 798 Evans Hall \#3840, Berkeley, CA 94720-3840, is published twelve times a year. Periodical rate postage paid at Berkeley, CA 94704, and additional mailing offices. POSTMASTER: send address changes to Pacific Journal of Mathematics, P.O. Box 4163, Berkeley, CA 94704-0163.

PJM peer review and production are managed by EditFLOW ${ }^{\circledR}$ from Mathematical Sciences Publishers.

\section{PUBLISHED BY}

mathematical sciences publishers

nonprofit scientific publishing

http://msp.org/

(C) 2013 Mathematical Sciences Publishers 


\section{PACIFIC JOURNAL OF MATHEMATICS}

Volume $265 \quad$ No. $1 \quad$ September 2013

Genus-two Goeritz groups of lens spaces

SANGBUM CHO

A compact embedding theorem for generalized Sobolev spaces

SENG-KeE ChUa, SCOTT Rodney and Richard L. WheEden

Partial integrability of almost complex structures and the existence of

solutions for quasilinear Cauchy-Riemann equations

CHONG-KYU HAN and JONG-DO PARK

An overdetermined problem in potential theory

DMITRY KHAVINSON, ERIK LUNDBERG and RAZVAN TEODORESCU

Quasisymmetric homeomorphisms on reducible Carnot groups

XIANGDONG XIE

Capillarity and Archimedes' principle

JOHN MCCUAN and RAY TREINEN

Generalized eigenvalue problems of nonhomogeneous elliptic operators and their application

DUmitru Motreanu and Mieko TANaKa

Weighted Ricci curvature estimates for Hilbert and Funk geometries

SHIN-ICHI OHTA

On generalized weighted Hilbert matrices

EMMANUEL PREISSMANN and OLIVIER LÉVÊQUE

Unique prime decomposition results for factors coming from wreath product 221 groups

J. OWEN SizEMORE and ADAM WinCHESTER

On volume growth of gradient steady Ricci solitons

Guofang Wei and Peng Wu

Classification of moduli spaces of arrangements of nine projective lines

FEI YE 